\title{
The role of the $17 q 21$ genotype in the prevention of early childhood asthma and recurrent wheeze by vitamin D
}

\author{
Rachel S. Kelly (1) ${ }^{1,2,7}$, Bo L. Chawes ${ }^{3,7}$, Feng Guo (1) ${ }^{1,2}$, Li Zhang ${ }^{1,4}$, \\ Kevin Blighe ${ }^{1}$, Augusto A. Litonjua $\mathbb{0}^{5}$, Benjamin A. Raby ${ }^{1,2,6}$, Bruce D. Levy ${ }^{2,6}$, \\ Daniela Rago ${ }^{3}$, Jakob Stokholm ${ }^{3}$, Klaus Bønnelykke ${ }^{3}$, Hans Bisgaard ${ }^{3}$, \\ Xiaobo Zhou ${ }^{1,2}$, Jessica A. Lasky-Su ${ }^{1,2,8}$ and Scott T. Weiss ${ }^{1,2,8}$
}

Affiliations: ${ }^{1}$ Channing Division of Network Medicine, Dept of Medicine, Brigham and Women's Hospital, Boston, MA, USA. ${ }^{2}$ Harvard Medical School, Boston, MA, USA. ${ }^{3}$ COPSAC, Copenhagen Prospective Studies on Asthma in Childhood, Herlev and Gentofte Hospital, University of Copenhagen, Copenhagen, Denmark. ${ }^{4}$ Dept of Integrated Traditional Chinese and Western Medicine, West China Hospital, Sichuan University, Chengdu, PR China. ${ }^{5}$ Pulmonary Division, Dept of Pediatrics, University of Rochester School of Medicine and Dentistry, Rochester, NY, USA. 'Pulmonary and Critical Care Medicine, Dept of Medicine, Brigham and Women's Hospital, Boston, MA, USA. ${ }^{7}$ Co-first authors. ${ }^{8}$ Co-senior authors.

Correspondence: Scott T. Weiss, Channing Division of Network Medicine, Dept of Medicine, Brigham and Women's Hospital, 181 Longwood Avenue, Boston, MA 02115, USA. E-mail: scott.weissdachanning.harvard.edu

@ERSpublications

Genetic variation in the 17 q21 locus and vitamin D play a role in asthma risk and, as demonstrated for the first time in a human population, sphingolipid biosynthesis may partially underlie these relationships http://bit.ly/2ZbIpsp

Cite this article as: Kelly RS, Chawes BL, Guo F, et al. The role of the $17 \mathrm{q} 21$ genotype in the prevention of early childhood asthma and recurrent wheeze by vitamin D. Eur Respir J 2019; 54: 1900761 [https://doi. org/10.1183/13993003.00761-2019].

ABSTRACT Evidence suggests vitamin D has preventive potential in asthma; however, not all children benefit from this intervention. This study aimed to investigate whether variation in the functional 17q21 single nucleotide polymorphism rs12936231 affects the preventive potential of vitamin D against asthma.

A combined secondary analysis of two randomised controlled trials of prenatal vitamin D supplementation for the prevention of asthma in offspring (Vitamin D Antenatal Asthma Reduction Trial (VDAART) and Copenhagen Prospective Studies on Asthma in Childhood 2010 (COPSAC 2010$)$ ) was performed, stratifying by genotype and integrating metabolite data to explore underlying mechanisms.

The protective effect of vitamin $\mathrm{D}$ on asthma/wheeze was evident among children with the low-risk rs12936231 GG genotype (hazard ratio (HR) 0.49, 95\% CI 0.26-0.94, p=0.032) but not the high-risk CC genotype (HR 1.08, 95\% CI 0.69-1.69, p=0.751). In VDAART, in the GG genotype vitamin D supplementation was associated with increased plasma levels of sphingolipids, including sphingosine-1phosphate ( $\beta$ 0.022, 95\% CI 0.001-0.044, $\mathrm{p}=0.038$ ), but this was not evident with the CC genotype, known to be associated with increased expression of ORMDL3 in bronchial epithelial cells. Sphingolipid levels were associated with decreased risk of asthma/wheeze, and there was evidence of interactions between

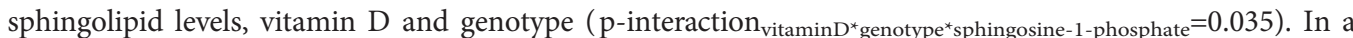
cellular model, there was a significant difference in the induction of sphingosine-1-phosphate by vitamin $\mathrm{D}$ between a control human bronchial epithelial cell line and a cell line overexpressing ORMDL3 $(\mathrm{p}=0.002)$.

Results suggest prenatal vitamin D supplementation may reduce the risk of early childhood asthma/ wheeze via alterations of sphingolipid metabolism dependent on the 17q21 genotype. 


\section{Introduction}

Asthma is hypothesised to emerge from gene-environment interactions disturbing fetal developmental processes in utero [1-3]. Therefore, novel preventive methods targeted at pregnant women may help alleviate the burden of asthma.

We recently conducted two independent randomised clinical trials (RCTs) of prenatal vitamin D supplementation for the reduction of asthma among offspring: the Vitamin D Antenatal Asthma Reduction Trial (VDAART) [4] and the Copenhagen Prospective Studies on Asthma in Childhood 2010 $\left(\mathrm{COPSAC}_{2010}\right)$ [5]. A combined analysis of the two trials demonstrated a significant $25 \%$ reduction in risk [6]. However, not all children benefited from the intervention and we hypothesise that the child's underlying genetic susceptibility to asthma may have attenuated the impact of vitamin D supplementation on asthma development.

Chromosomal region $17 q 21$ is the most replicated childhood asthma locus [7, 8]. The high-risk genotype defines an early-onset asthma phenotype with recurrent wheeze [9] that can be modulated by environmental exposures $[1,2,10]$. Expression of ORMDL3 at the $17 \mathrm{q} 21$ locus is thought to confer an increased risk of asthma, in part through inhibition of the serine palmitoyltransferase (SPT) enzyme. SPT catalyses the first step in the synthesis of sphingolipids: the condensation of serine and palmitoyl coenzyme A to produce 3-ketodihydrosphingosine [11]. It is therefore a key regulator of sphingolipid levels, which have been associated with bronchial reactivity and asthma in mechanistic studies [12-14]. However, human studies investigating whether ORMDL3-regulated sphingolipid pathways contribute to the pathogenesis of asthma are lacking [15].

In this study, we explored the hypothesis that genetic variation in the functional single nucleotide polymorphism (SNP) rs12936231 affected the outcome of our prenatal vitamin D trials, through alterations of sphingolipid metabolism. We focused on this SNP in particular because it has been denoted as the strongest candidate for a functional SNP within 17q21 [16], it is known to influence the expression of ORMDL3 in multiple cell types [16, 17], it has been repeatedly associated with asthma [12] and it has links to vitamin $\mathrm{D}$ in the literature [18]. We conducted this study in the VDAART and COPSAC 2010 populations, utilising genotype data, sphingolipid metabolite levels and cell line studies.

\section{Methods}

\section{Study populations}

The study populations have been described in detail previously $[19,20]$ and the trial protocols [4-6] are registered with ClinicalTrials.gov as NCT00920621 (VDAART) and NCT00856947 (COPSAC 2010$)$. In brief, both studies recruited pregnant women (VDAART: 10-18 weeks; COPSAC $_{2010}$ : 22-26 weeks) and randomised them to daily vitamin $\mathrm{D}_{3}$ (VDAART: $4000 \mathrm{IU}$; COPSAC 2010 : $2400 \mathrm{IU}$ ) or placebo until delivery. All women additionally received a daily multivitamin containing $400 \mathrm{IU}$ vitamin $\mathrm{D}_{3}$. Written and oral consent, including for secondary analyses, were obtained from both cohorts (full details in supplementary methods).

\section{Data collection}

In both cohorts, children were followed for incident asthma/recurrent wheeze (defined in supplementary methods) and genotyped for the $17 \mathrm{q} 21$ rs12936231 SNP with the Illumina Infinium HumanOmniExpressExome Bead chip (San Diego, CA, USA). For a subset of children, metabolomic profiling was performed by Metabolon, Inc. (NC, USA) on plasma samples (VDAART: ages 1 and 3; COPSAC $_{2010}$ : age 6 months) (supplementary methods). We extracted the five available metabolites from the sphingolipid biosynthesis pathway: sphingosine-1-phosphate (shown to be one of the most important sphingolipid metabolites for airway hyperresponsiveness, mast cell activation and inflammation in mechanistic asthma models $[13,14,21])$, sphinganine, sphinganine-1-phosphate, phosphoethanolamine and sphingosine.

\section{Statistics}

Differences between the vitamin D and placebo arms were assessed using the Chi-squared test. In each 17q21 genotype strata (GG, GC, CC), the effect of prenatal vitamin D supplementation on asthma/recurrent

This article has supplementary material available from erj.ersjournals.com

This is a combined secondary analysis of two trials with clinicaltrials.gov identifiers NCT00920621 (VDAART) and NCT00856947 (COPSAC 2010 ). Because both COPSAC $_{2010}$ and VDAART are ongoing, full phenotype and genetic data will not be available until after the studies have been completed.

Received: 15 April 2019 | Accepted after revision: 18 July 2019 
wheeze from age 0 to 3 years was analysed by event-time models using Cox proportional hazards regression. Analyses were conducted independently in each cohort then combined using a fixed-effects meta-analysis model, weighting estimates by the inverse of the estimate variance. The heterogeneity between the two studies was tested with a Cochran Q test and quantified using $\mathrm{I}^{2}$ statistics.

To explore the role of sphingolipid metabolism in the relationship between 17q21 genotype and asthma/recurrent wheeze, we determined the association between the vitamin $\mathrm{D}$ intervention and sphingolipid peak intensities at ages 1 and 3 years using linear regression models, stratified by 17q21 genotype. In order to account for the 203 subjects with measures of sphingolipids at both ages, we additionally performed combined analyses using a linear mixed model with a random intercept. We then constructed multivariable regression models to test for interactions between rs12936231 genotype, prenatal vitamin D supplementation and sphingolipid metabolite level in the risk of asthma/recurrent wheeze by age 3 years.

\section{Assessment of sphingosine-1-phosphate concentration in an ORMDL3-overexpressing cell line}

To determine whether there are functional implications of vitamin D treatment on sphingosine-1phosphate levels when ORMDL3 is overexpressed, we used lentiviral-based infection to establish a human bronchial epithelial cell line, using $16 \mathrm{HBE}$ cells, that overexpressed ORMDL3. Supplementary figure E1 demonstrates the efficiency of the overexpression of ORMDL3, as demonstrated by both reverse transcriptase PCR at the mRNA level and by Western blotting at the protein level. We treated 16HBE cells with or without overexpression of ORMDL3 with $1 \alpha, 25$-vitamin $\mathrm{D}_{3}$ (Sigma-Aldrich, St Louis, MO, USA) at varying concentrations $(0.0,0.1$ and $1.0 \mathrm{nM})$ for $10 \mathrm{~h}$, then measured cytoplasmic sphingosine-1-phosphate levels by ELISA (MyBioSource, San Diego, CA, USA). Three biological replicates were performed at each concentration. An unpaired t-test was used to compare induced sphingosine-1-phosphate levels (ng. $\mathrm{mL}^{-1}$ ) in the control cell line and in the cell line overexpressing ORMDL3 (supplementary methods).

All statistical analyses were conducted using R v.3.2.3 (R Foundation for Statistical Computing, Vienna, Austria) and the packages "survival", "survminer", "meta" and "AUCRF". All hypothesis tests were two sided and a confidence level of $95 \%$ was employed.

\section{Results}

\section{Baseline characteristics}

The study populations for each of the conducted analyses are described in supplementary table E1. The primary analyses in both studies were based on those with genotype data and complete follow-up for asthma/recurrent wheeze until age 3 years (table 1). In VDAART, the genotype distribution was CC $\mathrm{n}=171$ (27.7\%), GC $\mathrm{n}=313$ (50.6\%) and GG $\mathrm{n}=134$ (21.7\%), corresponding to a minor allele frequency (MAF) of 0.47 ( $\mathrm{G}$ allele) and Hardy-Weinberg equilibrium (HWE) $\mathrm{p}=0.72$. In $\mathrm{COPSAC}_{2010}$, it was CC $\mathrm{n}=121$ (23.4\%), GC $\mathrm{n}=259$ (50.1\%) and GG $\mathrm{n}=137$ (26.5\%), corresponding to a MAF of 0.52 and HWE $\mathrm{p}=0.98$. There was no difference in the distribution of genotype, sex, asthma/wheeze status or race between the vitamin $\mathrm{D}$ and placebo arms in either VDAART (vitamin $\mathrm{D} n=311$, placebo $\mathrm{n}=307$ ) or COPSAC 2010 (vitamin $D n=261$, placebo $n=256$ ) and the relative proportions were representative of the two parent studies $[4,5]$. Serum vitamin D levels at age 3 years were available for VDAART only; although the point estimates were higher among the children whose mothers received the intervention, this difference was not significant. Similarly, there were no significant differences in serum vitamin D levels in VDAART between the placebo and intervention group when stratified by genotype ( $p>0.1$ for all comparisons). In COPSAC $_{2010}$ a proportion of mothers additionally received n-3 long-chain polyunsaturated fatty acids via fish-oil capsules during pregnancy, but we determined there was no significant difference in genotype distribution among the four intervention groups of vitamin D only, fish oil only, vitamin D and fish oil, and placebo only (supplementary table E2).

\section{Vitamin D, 17q21 genotype and development of asthma/recurrent wheeze}

The results from both trials suggested that the effect of the prenatal vitamin $\mathrm{D}$ supplementation on the development of asthma/recurrent wheeze from ages 0 to 3 years was dependent on the child's $17 \mathrm{q} 21$ genotype, with an increasingly protective effect of vitamin $\mathrm{D}$ with decreasing number of $\mathrm{C}$ risk alleles (supplementary figure E2). The high-dose vitamin D intervention in the rs12936231 genotype strata in VDAART resulted in the following hazard ratios for developing asthma/recurrent wheeze: for CC, HR 1.07 (95\% CI 0.59-1.95); for GC, HR 0.75 (95\% CI 0.50-1.11); and for GG, HR 0.51 (95\% CI 0.21-1.19). In COPSAC $_{2010}$, the observed pattern was very similar: for CC, HR 1.08 (95\% CI 0.55-2.15); for GC, HR 0.73 (95\% CI 0.39-1.37); and for GG, HR 0.48 (95\% CI 0.18-1.27).

A combined analysis of the two trials confirmed that the high-dose vitamin D intervention conferred a $50 \%$ reduced risk of developing asthma/recurrent wheeze by age 3 years in children with the low-risk GG 
TABLE 1 Baseline characteristics of the participants from the VDAART and COPSAC 2010 prenatal vitamin D trials with genotype data stratified by intervention

\begin{tabular}{|c|c|c|c|c|c|c|}
\hline Characteristic & \multicolumn{3}{|c|}{ VDAART $^{\#}$} & \multicolumn{3}{|c|}{$\operatorname{COPSAC}_{2010}$ ๆ } \\
\hline $\begin{array}{l}\text { Subjects n } \\
\text { rs12936231 }\end{array}$ & 307 & 311 & & 256 & 261 & \\
\hline $\mathrm{CC}$ & $78(25.4)$ & 93 (29.9) & 0.454 & 59 (23) & 62 (23.8) & 0.820 \\
\hline GC & 161 (52.4) & 152 (48.9) & & $126(49.2)$ & $133(51)$ & \\
\hline GG & $68(22.1)$ & 66 (21.2) & & $71(27.7)$ & 66 (25.3) & \\
\hline Yes & $92(30)$ & $76(24.4)$ & & 50 (19.5) & $42(16.1)$ & \\
\hline \multicolumn{7}{|l|}{ Sex } \\
\hline Male & 164 (53.4) & 157 (50.5) & 0.515 & $128(50)$ & $141(54)$ & 0.408 \\
\hline Female & $143(46.6)$ & 154 (49.5) & & $128(50)$ & $120(46)$ & \\
\hline \multicolumn{7}{|l|}{ Race } \\
\hline Black & $146(47.6)$ & 144 (46.3) & 0.359 & & & \\
\hline
\end{tabular}

Data are presented as $\mathrm{n}(\%)$ or mean $\pm \mathrm{SD}$, unless otherwise indicated. VDAART: Vitamin D Antenatal Asthma Reduction Trial; $\mathrm{COPSAC}_{2010}$ : Copenhagen Prospective Studies on Asthma in Childhood 2010. \#: the total VDAART population was 806 children, data for 618 were included, the dose was $4400 \mathrm{IU}$ daily in the intervention arm versus $400 \mathrm{IU}$ in the placebo arm and was initiated in weeks $10-18$; ${ }^{\text {?: }}$ the total COPSAC 2010 population was 581, data for 517 were included, the dose was 2800 IU daily in the intervention arm versus 400 IU in the placebo arm and was initiated in weeks $22-26 ;{ }^{+}$: available for VDAART only.

genotype (fixed-effects HR 0.49 , 95\% CI 0.26-0.94). This protective effect decreased with increasing number of risk alleles (for GC, HR 0.74, 95\% CI 0.53-1.04; for CC, HR 1.08, 95\% CI 0.69-1.69) (table 2). These findings also held true when assuming the $\mathrm{G}$ allele is dominant (supplementary table E3).

\section{Vitamin D and sphingolipid metabolism}

Metabolomic profiling of plasma was available in a subset of the VDAART children in the samples extracted at age 1 year $(n=413)$ and age 3 years $(n=353$, including 203 of the children with a 1-year sample) (supplementary table E1). In the linear regression models, there was a consistently positive association between prenatal vitamin D intervention and higher levels of the five sphingolipids among children with the GG genotype. This association reached significance in the mixed models including all samples for sphinganine-1-phosphate ( $\beta$ 0.040, 95\% CI 0.002-0.078, $\mathrm{p}=0.038)$ and sphingosine-1phosphate $(\beta 0.022,95 \%$ CI $0.001-0.044, p=0.038)$, and was approaching significance $(p<0.1)$ for sphinganine, sphingosine and phosphoethanolamine. In contrast, there was no observed increase in sphingolipid levels associated with the vitamin D intervention in those children with the high-risk CC genotype (table 3). However, these findings were not recapitulated in the 6-month samples from 441 children from COPSAC $_{2010}$ (supplementary table E4). Combined analyses with VDAART were not conducted owing to differences in sample collection age.

TABLE 2 Effect of prenatal vitamin D supplementation versus placebo on the development of asthma/persistent wheeze from 0 to 3 years in the VDAART and COPSAC 2010 trials stratified by genotype of the $17 q 21$ functional SNP rs 12936231

\begin{tabular}{|c|c|c|c|c|c|c|c|c|}
\hline \multirow[t]{2}{*}{ rs12936231 strata } & \multicolumn{3}{|c|}{ VDAART $^{\#}$} & \multicolumn{3}{|c|}{$\operatorname{COPSAC}_{2010}$ " } & \multicolumn{2}{|c|}{ Combined analyses } \\
\hline & Total/cases $\mathbf{n}$ & $\operatorname{HR}(95 \% \mathrm{CI})$ & p-value & Total/cases $\mathbf{n}$ & $\operatorname{HR}(95 \% \mathrm{CI})$ & p-value & $\operatorname{HR}^{+}(95 \% \mathrm{CI})$ & p-value \\
\hline GG & $134 / 23$ & $0.51(0.21-1.19)$ & 0.119 & $137 / 19$ & $0.48(0.18-1.27)$ & 0.140 & $0.49(0.26-0.94)$ & 0.032 \\
\hline CC & $171 / 44$ & $1.07(0.59-1.95)$ & 0.821 & $121 / 33$ & $1.08(0.55-2.15)$ & 0.822 & $1.08(0.69-1.69)$ & 0.751 \\
\hline
\end{tabular}

Cases refer to the number of children with asthma/persistent wheeze at from age 0 to 3 years. VDAART: Vitamin D Antenatal Asthma Reduction Trial; COPSAC 2010: Copenhagen Prospective Studies on Asthma in Childhood 2010; SNP: single nucleotide polymorphism. ${ }^{\#}$ : $\mathrm{n}=618$; ก: $n=517 ;^{+}$: fixed-effects meta-analysis HR combining VDAART and COPSAC 2010. 
TABLE 3 Association between blood peak intensity of five key sphingolipid metabolites and vitamin D intervention in VDAART children stratified by 17q21 genotype

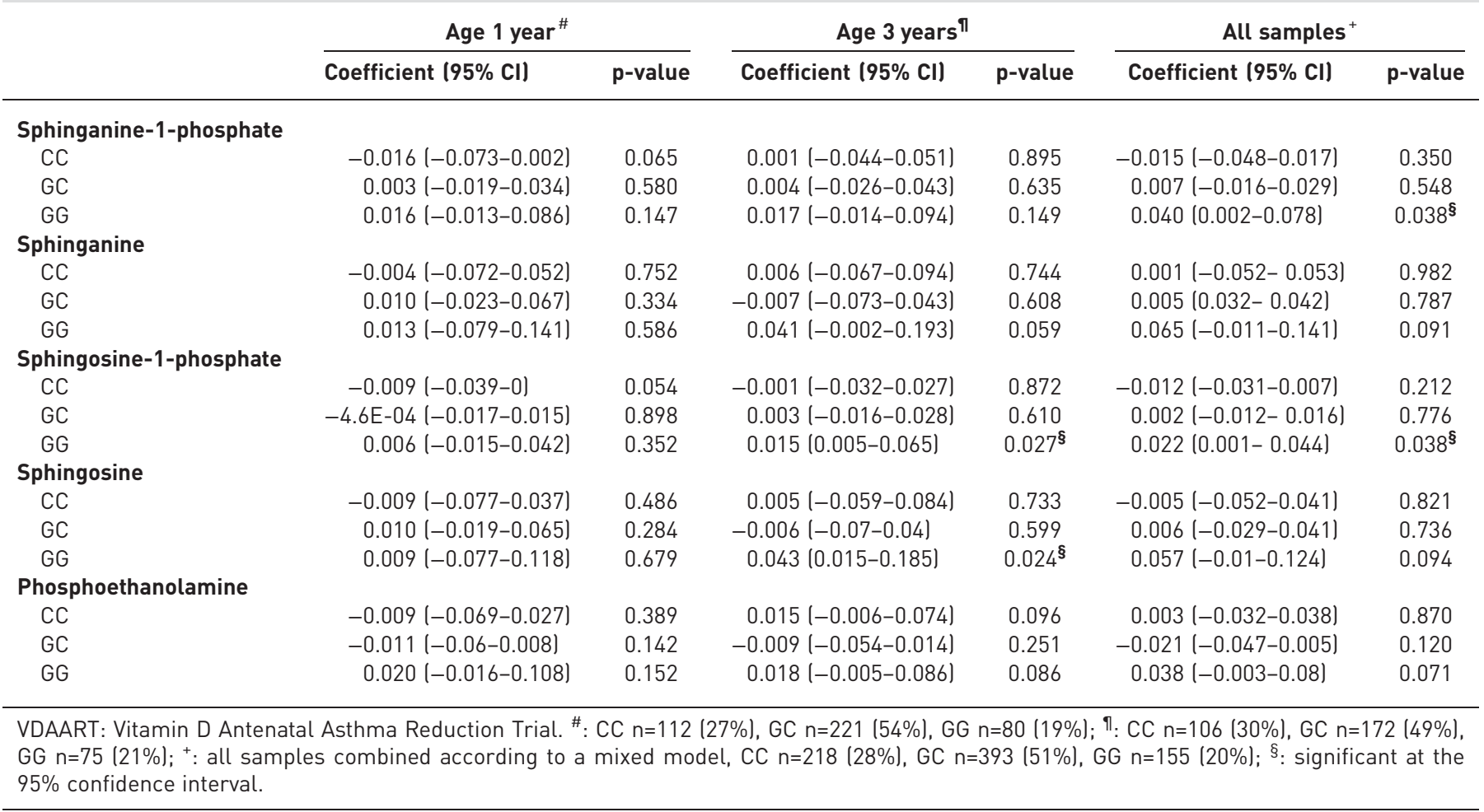

Vitamin D, sphingolipid metabolism, 17q21 genotype and development of asthma/recurrent wheeze

In VDAART, sphingosine-1-phosphate, phosphoethanolamine and sphinganine-1-phosphate demonstrated a significant $(\mathrm{p}<0.05)$ or approaching significant $(\mathrm{p}<0.1)$ three-way interaction between the vitamin $\mathrm{D}$ intervention, rs12936231 genotype and metabolite peak intensity in the risk of asthma/recurrent wheeze at ages 1 and 3 years. This suggests that sphingolipid metabolites and the rs12936231 genotype may jointly influence the effect of vitamin $\mathrm{D}$ on asthma risk (supplementary table E5). However, significant interactions were not noted in the 6-month samples from COPSAC 2010 (supplementary table E6).

\section{Sensitivity analysis}

Race

Sensitivity analyses were run to account for the potential influence of race in VDAART. Genotype frequencies were very similar in the African American ( $\mathrm{n}=290$ children) and Caucasian $(\mathrm{n}=200)$ populations, although they deviated slightly in the "Other" category which included Asian and Native Hawaiian children $(n=128)$ (supplementary table E7). Cox proportional regression models were rerun and stratified by race, and then the results were combined using a meta-analysis model. Owing to small numbers, the "Other" and "Caucasian" categories were combined. The combined results were supportive of the conclusion that the effect of prenatal vitamin D supplementation on the development of asthma/recurrent wheeze was modified by the $17 \mathrm{q} 21$ genotype (supplementary table E8). There was no protective effect of the vitamin D intervention in children with the high-risk CC genotype (HR 1.05, 95\% CI 0.58-1.92), while there was a trend towards an increasingly protective effect with a decreasing number of risk alleles (for GC, HR 0.76, 95\% CI 0.51-1.14; for GG, HR 0.53, 95\% CI 0.22-1.29). However, it should be noted that these results appeared to be largely driven by the data from African American children.

We additionally reran the regression models and interactions models for the sphingolipid analyses, stratifying by race category. While the overall pattern of results in terms of genotype and direction of effect held true across all populations for both the vitamin D supplementation-metabolite regression model (supplementary table E9) and the multivariable interaction model (supplementary table E10), there was some evidence that these results were being driven by data from children in the African American and Other populations. 
Fish-oil supplementation

To reflect the fact that a proportion of the included mothers in COPSAC $_{2010}$ were also receiving fish-oil supplementation throughout pregnancy, we reran the analyses excluding the 256 children of these women. When comparing the 128 children whose mothers received only vitamin D with the 133 whose mothers received placebo only, there remained evidence of a trend for an increasing protective effect of vitamin $\mathrm{D}$ supplementation with decreasing number of $\mathrm{C}$ alleles. Furthermore, when combined with the VDAART population, the decreased risk in the GG strata was borderline significant with a similar magnitude to when the full $\mathrm{COPSAC}_{2010}$ population was included (HR 0.49, 95\% CI 0.24-1.02, p=0.055), while there was no evidence of a protective effect in the CC strata (HR 1.00, 95\% CI 0.60-1.66, p=0.999) (supplementary table E11).

We reran the sphingolipid analyses excluding the children whose mothers had received fish-oil supplementation throughout pregnancy. The results were largely unchanged for the regression models (supplementary table E12) in terms of direction of effect and significance level. Similarly, the results of the three-way interaction population were consistent when the fish-oil supplementation subgroup was removed (supplementary table E13). Taken together, these results suggest that concurrent fish-oil supplementation was not affecting the results in the COPSAC 2010 population with regards to the genotype-specific protective effect of vitamin D supplementation and its relationship with sphingolipids.

\section{Safety}

There was no significant difference in the rates of severe adverse events between the trial arms in either study $[4,5]$.

Assessment of sphingosine-1-phosphate concentration in human bronchial epithelial cells Given the observed interaction between vitamin D treatment, sphingosine-1-phosphate levels and the rs12936231 genotype in human subjects, we then explored the relationship between ORMDL3 expression and levels of sphingosine-1-phosphate in response to vitamin D treatment in cellular models. We performed three biological replicates at three concentrations of $1 \alpha, 25$-vitamin $\mathrm{D}_{3}$ : $0.0 \mathrm{nM}, 0.1 \mathrm{nM}$ and $1.0 \mathrm{nM}$. We then compared the mean level of induced sphingosine-1-phosphate $\left(\mathrm{ng} \cdot \mathrm{mL}^{-1}\right)$ across the three replicates between the control and the ORMDL3-overexpressing cell line for each concentration. We found no significant difference in the levels of induced sphingosine-1-phosphate between the control and the ORMDL3-overexpressing cell line when treated with $0.0 \mathrm{nM}$ and $0.1 \mathrm{nM}$ of $1 \alpha, 25$-vitamin $\mathrm{D}_{3}$. However, there was a significant difference $(\mathrm{p}=0.002)$ between the control (mean of three replicates $62.3 \mathrm{ng} \cdot \mathrm{mL}^{-1}$ ) and the ORMDL3 line (mean of three replicates $53.0 \mathrm{ng} \cdot \mathrm{mL}^{-1}$ ) when treated with $1.0 \mathrm{nM}$ (figure 1). This suggests that higher expression of ORMDL3, generally associated with risk CC genotype at rs12936231 [17], leads to reduced induction of sphingosine-1-phosphate by vitamin D in human bronchial epithelial cells.

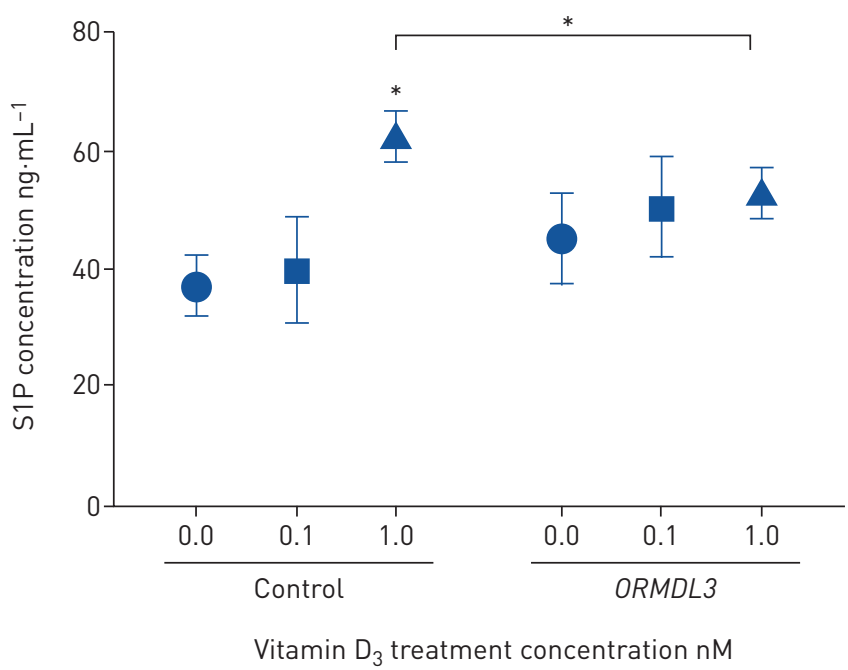

FIGURE 1 Measurements of sphingosine-1-phosphate (S1P) levels in 16HBE cells after vitamin $\mathrm{D}_{3}$ treatment. $16 \mathrm{HBE}$ human bronchial epithelial cells and ORMDL3-overexpressing stable 16HBE cells were treated with vitamin $D_{3}$ at three concentrations $(0.0,0.1$ and $1.0 \mathrm{nM})$ for $10 \mathrm{~h}$. Levels of S1P in cells were measured by ELISA. Mean \pm SD shown for three independent experiments in ELISA assay. *: $p<0.05$, unpaired t-test. 


\section{Discussion}

An increasing body of evidence now supports the preventive and protective potential of vitamin $\mathrm{D}$ on asthma and its symptoms [22]. This study suggests that the preventive actions of prenatal vitamin D supplementation on early childhood asthma/wheeze during the first 3 years of life may be modified by genetic variants in $17 \mathrm{q} 21$, a crucial asthma genome-wide association study locus. Children with the low-risk GG genotype in rs12936231 whose mothers were supplemented with vitamin D had a $50 \%$ reduced risk of asthma, whereas there was no protective effect in children with the high-risk CC genotype. Based on our exploratory sphingolipid and functional work, we hypothesise that for a child with the non-risk GG genotype, prenatal vitamin D supplementation increases sphingolipid metabolism, and subsequently the levels of downstream sphingolipids that are protective against asthma/recurrent wheeze. Conversely, for a child with the high-risk CC genotype, increased expression of ORMDL3, known to inhibit a rate-limiting enzyme for de novo sphingolipid biosynthesis, SPT [23], modifies the relationship

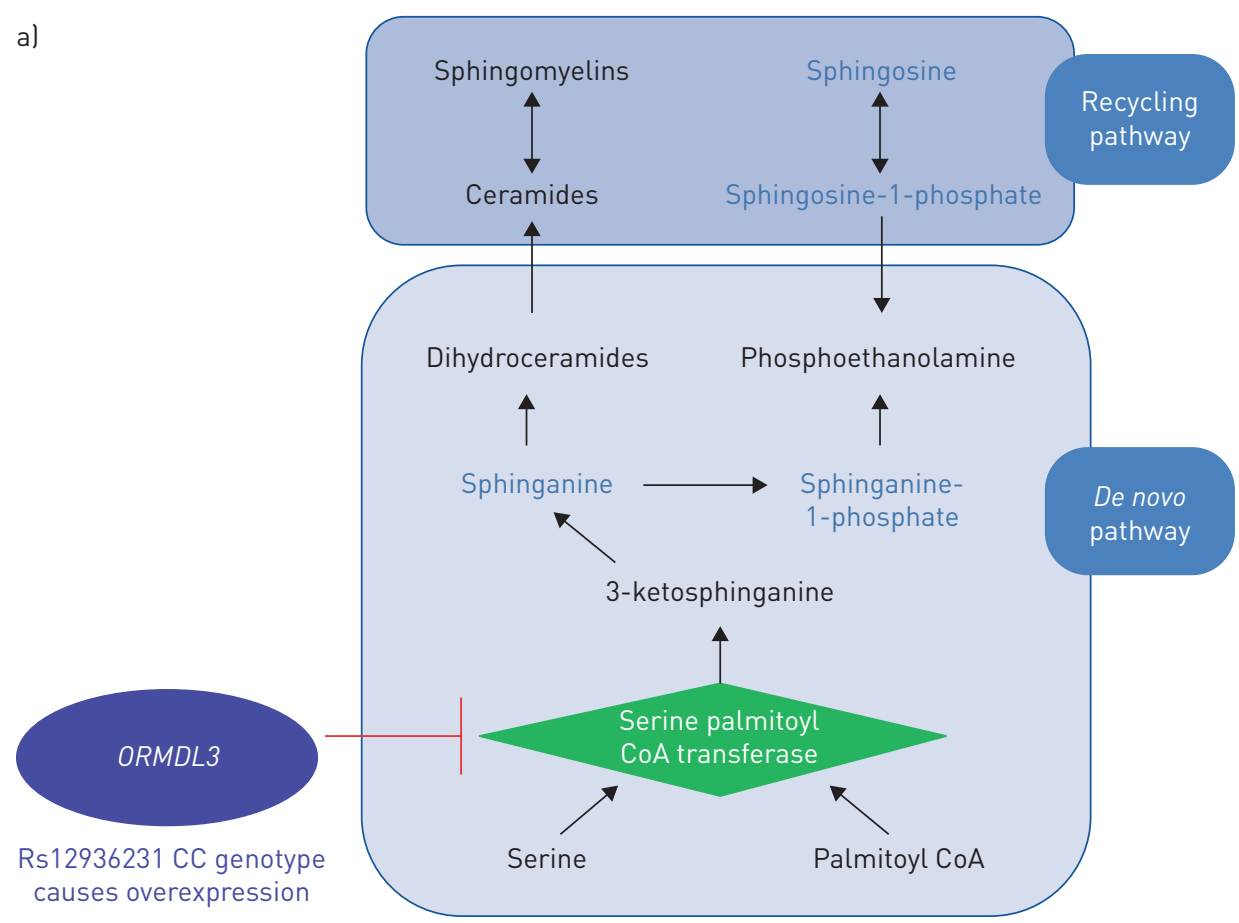

b)

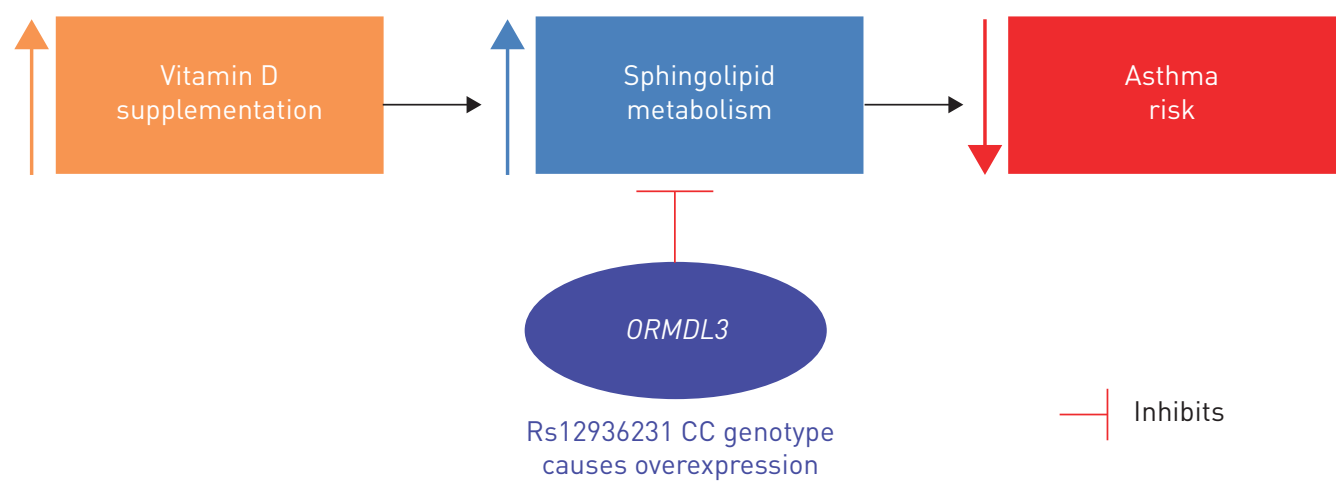

FIGURE 2 Hypothesised mechanism. a) Sphingolipid metabolism: serine palmitoyltransferase catalyses a crucial reaction in the production of sphingolipids. ORMDL3 inhibits the action of serine palmitoyltransferase and therefore the de novo pathway production of sphingolipids. Expression of ORMDL3 is increased by the CC genotype at rs12936231, leading to increased inhibition of sphingolipid production. b) Hypothesised mechanism: prenatal vitamin D supplementation increases the production of sphingolipids in offspring via the sphingolipid metabolism pathway, resulting in a decreased risk of asthma. When ORMDL 3 is overexpressed the sphingolipid metabolism pathway is inhibited, sphingolipid production is not increased and there is no protective effect of vitamin D on asthma risk. CoA: coenzyme A. 
between vitamin $\mathrm{D}$ and sphingolipid synthesis, and thus may render the vitamin $\mathrm{D}$ intervention ineffective at reducing asthma/recurrent wheeze risk (figure 2).

17 q21 was first discovered as a childhood asthma susceptibility locus in 2007 , when genetic variants were shown to regulate transcription of the ORMDL3 gene in lymphoblastoid cell lines [24]. In this study we focused on a specific SNP in this region, rs12936231, a functional variant located in intron 4 of ZPBP2 that independently controls ORMDL3 expression [12]. rs12936231 alters the CTCF binding motif with a G to $\mathrm{C}$ change, to the point where it is almost completely lost in the ZPBP2 intronic region for subjects carrying the asthma-risk $C$ allele $[12,25]$. Instead, it switches the binding site of CTCF to the ORMDL3 intronic region, which is hypothesised to alter the three-dimensional architecture of the 17q21 locus, favouring enhanced transcription of $\operatorname{ORMDL3}[16,12]$. The asthma-associated C allele of this SNP is therefore associated with downregulation of sphingolipid metabolism via increased expression of ORMDL3 and inhibition of SPT [25]. The link between sphingolipid metabolism and asthma is further supported by mouse studies showing that decreased sphingolipid synthesis in lung epithelial tissue [14] and SPT knockouts [21] both associate with increased airway hyperresponsiveness and inflammation [13], and that sphingolipids play an important role in the maturing postnatal lung [26]. Interestingly, experimental studies have shown that vitamin D metabolites are capable of activating the sphingolipid pathway [27, 28], and vitamin D has been shown to alter the recruitment of CTCF [18], making rs12936231 an SNP of particular interest for this study. rs12936231 is also unique in that it has been shown to impact the function of not only immune cells but also multiple other cell types that are potentially involved in asthma pathogenesis [16]. Taken together, it is biologically plausible that prenatal vitamin D supplementation acts to reduce the risk of childhood asthma in part through 17q21-dependent sphingolipid metabolism, and that rs12936231 is a particularly informative SNP to study in this regard.

We observed a clear allele-additive modifying effect, in two independent cohorts, of rs12936231, with a decreasing effect of the vitamin D intervention on asthma/recurrent wheeze with an increasing number of risk alleles. In VDAART, we then determined that prenatal vitamin D supplementation resulted in increased levels of key sphingolipids in offspring with the rs12936231 GG or GC genotype, but not those with the CC risk genotype. Furthermore, we present some evidence of significant interactions between prenatal vitamin D intervention with offspring genotype and sphingolipid metabolites on the risk of asthma/recurrent wheeze, again suggesting that the effect of prenatal supplementation may be modified by these factors. Additional supporting evidence came from cellular models. Genetic overexpression of ORMDL3 led to decreased induction of sphingosine-1-phosphate in response to vitamin $\mathrm{D}$ treatment. This suggests that ORMDL3 may modify the vitamin D-sphingosine-1-phosphate relationship. Nevertheless, we recognise that no cellular model can completely recapitulate the effects of prenatal vitamin D supplementation on the risk of asthma in offspring. Furthermore, it should be noted that in addition to human bronchial epithelial cells there are other sources of sphingosine-1-phosphate production.

Differences in the trial design and populations between the two cohorts represent one of the biggest limitations of this study. VDAART is a high-risk cohort recruiting parents with asthma/allergy, and includes a large group of African Americans in the trial, who have a higher risk of developing asthma and allergy [29]. In contrast, COPSAC $\mathrm{C}_{2010}$ is a population-based cohort which only genotyped Caucasian individuals. This is important because the 17q21-associated increased risk of childhood asthma and 17q21 MAFs vary on an SNP-level basis across ethnicities [25]. However, we observed very similar MAFs in VDAART and COPSAC ${ }_{2010}$ and sensitivity analyses demonstrated that the genotype results were not confounded by race. Despite the heterogeneity between the trials, we observed a nearly identical pattern of an increasing preventive effect of vitamin $\mathrm{D}$ by decreasing number of $17 \mathrm{q} 21$ risk alleles in both trials. It should also be noted that unlike VDAART, COPSAC 2010 had a randomisation within a cohort design; however, it has been stated that the findings from such studies are as valid as those from conventional RCT [30]; therefore, we are not concerned that this affected our results and conclusions regarding the influence of rs12936231.

We were not able to validate our exploratory sphingolipid findings from VDAART in the COPSAC 2010 population, which we believe may be due to underlying differences between the populations. Potentially the most important of these differences was the factorial design of COPSAC 2010 , whereby a proportion of mothers were additionally receiving daily prenatal supplementation of $n-3$ long-chain polyunsaturated fatty acids via fish-oil capsules [31]. There is some evidence that fish oil can alter sphingolipid metabolism and sphingolipid levels [32,33], which may explain the lack of difference in sphingolipid levels between the vitamin D and placebo groups. Therefore, we ran additional sensitivity analyses excluding the children of mothers who received fish oil throughout pregnancy. These analyses did not support our hypotheses that the lack of replication was due to the fish oil; however, we were somewhat limited by power in these analyses and we cannot discount that residual confounding from this additional supplementation may have influenced our COPSAC $_{2010}$ findings. Importantly, there were also substantial dietary differences 
between the two populations, with lower baseline levels of vitamin $\mathrm{D}$ and more vitamin $\mathrm{D}$-deficient mothers in the VDAART trial [6], which likely resulted in differing baseline sphingolipid levels. Finally, the timing of the blood sampling, the onset of supplementation and the daily dose of vitamin D differed between the two cohorts, which may again explain the lack of replication.

Based on our race-stratified sphingolipid findings in the VDAART population, in which we found that the significant results of interest were primarily driven by the non-Caucasian children, we suggest that race may have played a role in our inability to replicate the sphingolipid findings in COPSAC 2010 . We are not aware of evidence in the literature for a difference in sphingolipid metabolism by race; however, there is evidence to suggest that vitamin D metabolism may differ by race [34], which may help to explain the results of our race-stratified analyses. Consequently, although the genetic findings were of similar magnitude and reached statistical significance when combined, and we demonstrated that the COPSAC 2010 genetic findings were not driven by the fish-oil supplementation, further work is needed to explore the wider generalisability of the sphingolipid analyses.

We also note that we were limited by power in some of the cohort-specific stratified analyses, particularly with respect to the number of cases in the GG genotype strata. We note that for both cohorts the trend was the same, but it was only in the combined meta-analysis that the results reached significance. Furthermore, owing to the secondary and exploratory nature of these analyses, they should be considered hypothesis generating and a nominal p-value threshold was used to denote significance. Similarly, we did not account for multiple testing in our sphingolipid analysis; however, given the highly correlated nature of these metabolites and their existence within a single coordinated metabolic pathway, application of classical multiple testing corrections such as Bonferroni would be too stringent for such data. We also recognise that given the complexity of the $17 \mathrm{q} 21$ locus there are possibly other causal variants or genes that may influence risk. However, given the demonstrated independent functional nature of rs12936231 ORMDL3 expression [12, 16], and the role of ORMDL3 in sphingolipid biosynthesis [35], we consider these optimal for investigation.

Despite the limitations, this study was unique in our access to data from two independent prenatal vitamin $\mathrm{D}$ RCTs, which were aligned with respect to the predetermined endpoint of asthma/recurrent wheeze by age 3 years and having available genotyping. This enabled the discovery and replication of a $17 \mathrm{q} 21$ genotype-dependent effect of prenatal vitamin $\mathrm{D}$ intervention on asthma/recurrent wheeze. The sphingolipid data are a major advantage of this study and serve as an example of how this metabolomics, which provides a physiological "snapshot" of a biological system, may be useful for increasing the understanding of gene-environment interactions in health and disease. This approach enabled us to generate hypotheses regarding plausible underlying biological mechanisms [23], which we then explored in a cellular model.

We were unable to determine, within the confines of this study, whether the lack of preventive effect of vitamin $\mathrm{D}$ in children carrying the high-risk 17q21 genotype was due to our use of a dosage of vitamin D that was too low or was initiated too late in pregnancy or whether these children have a complete breakdown of the sphingolipid pathway rendering even high doses of vitamin D ineffective. We also recognise that vitamin $\mathrm{D}$ likely has other effects on fetal programming very early in pregnancy and may act to reduce the risk of asthma through immune modulations [36] and effects on lung organogenesis from branching morphogenesis throughout the alveolar stage of lung development [37, 38]. Furthermore, it has been previously noted that the effect of ORMLD3 overexpression on sphingolipid metabolism is dependent on both the extent of overexpression and the underlying physiological conditions [39]. Finally, we acknowledge that race may have influenced our findings, particularly with regard to the sphingolipid results. Additional work is required to explore these issues, specifically within a study with adequate sample size and power for the strata of interest.

\section{Conclusion}

This is the first study to demonstrate a link between vitamin D and the $17 \mathrm{q} 21$ locus in the development of childhood asthma and recurrent wheeze. Based on our exploratory analyses, we suggest that vitamin D acts to reduce the risk of asthma through an increase in sphingolipid metabolism, but that this pathway may be attenuated in those with key genetic variants in $17 \mathrm{q} 21$ that influence the expression of ORMDL3. These findings provide additional insights into the pathogenesis of childhood asthma and in particular the role of ORMDL3-regulated sphingolipid pathways [15].

Acknowledgements: The authors wish to thank the study participants of the VDAART and COPSAC 2010 trials and all those involved in collecting and managing the data for the parent studies. The results of the primary trials and a combined analysis of the two trials have been published previously. 
Author contributions: R.S. Kelly and B.L. Chawes performed the statistical analysis and wrote the first draft of the manuscript. Functional work was performed by F. Guo, L. Zhang and X. Zhou, with support from B.A. Raby and B.D. Levy. A.A. Litonjua and S.T. Weiss are the principal investigators of VDAART and H. Bisgaard is the principal investigator of COPSAC 2010 , and as such contributed to the overall concept and study design together with J.A. Lasky-Su; all four provided funding support. K. Blighe, D. Rago, J. Stokholm and K. Bønnelykke provided analytical support. All authors assisted with the editing of the final draft of the manuscript and provide their approval for its submission. The corresponding author had full access to the data and had final responsibility for the decision to submit for publication and takes full responsibility for the integrity of the data and the accuracy of the data analysis. No honorarium, grant or other form of payment was given to anyone to produce the manuscript.

Conflict of interest: R.S. Kelly has nothing to disclose. B.L. Chawes has nothing to disclose. F. Guo has nothing to disclose. L. Zhang has nothing to disclose. K. Blighe has nothing to disclose. A.A. Litonjua reports author royalties from UpToDate, Inc. B.A. Raby has nothing to disclose. B.D. Levy reports grants from National Institutes of Health and personal fees for consultancy from GossamerBio, Novartis, Pieris Pharmaceuticals, Sanofi and Teva, during the conduct of the study. D. Rago has nothing to disclose. J. Stokholm has nothing to disclose. K. Bønnelykke has nothing to disclose. H. Bisgaard has been a consultant for Chiesi and Boehringer Ingelheim. X. Zhou has nothing to disclose. J.A. Lasky-Su has nothing to disclose. S.T. Weiss reports that he is an author for UpToDate, principal investigator of several National Institutes of Health grants and an unpaid advisor to Novartis Pharmaceuticals.

Support statement: VDAART was supported by U01HL091528, 1R01HL123915-01 and 1R01HL123546-01A1 from the National Heart, Lung, and Blood Institute (NHLBI); and U54TR001012 from the National Centers for Advancing Translational Sciences. Metabolomic analyses and R.S. Kelly were supported by 5R01HL123915-05, 1R01HL141826-0 and W81XWH-17-1-0533. COPSAC 2010 was supported by The Lundbeck Foundation (R16-A1694); The Danish Ministry of Health (903516); Danish Council for Strategic Research (grant number 0603-00280B) and The Capital Region Research Foundation (full list www.copsac.com). B.L. Chawes was supported by a European Respiratory Society fellowship. The funding bodies played no role in the design or conduct of the study; collection, management, analysis or interpretation of the data; preparation, review or approval of the manuscript; nor decision to submit the manuscript for publication. Funding information for this article has been deposited with the Crossref Funder Registry.

\section{References}

1 Bouzigon E, Corda E, Aschard H, et al. Effect of 17q21 variants and smoking exposure in early-onset asthma. N Engl J Med 2008; 359: 1985-1994.

2 Çalışkan M, Bochkov YA, Kreiner-Møller E, et al. Rhinovirus wheezing illness and genetic risk of childhood-onset asthma. N Engl J Med 2013; 368: 1398-1407.

3 Renz H, Conrad M, Brand S, et al. Allergic diseases, gene-environment interactions. Allergy 2011; 66: 10-12.

4 Litonjua AA, Carey VJ, Laranjo N, et al. Effect of prenatal supplementation with vitamin D on asthma or recurrent wheezing in offspring by age 3 years: The VDAART randomized clinical trial. JAMA 2016; 315: 362-370.

5 Chawes BL, Bønnelykke K, Stokholm J, et al. Effect of vitamin D3 supplementation during pregnancy on risk of persistent wheeze in the offspring: a randomized clinical trial. JAMA 2016; 315: 353-361.

6 Wolsk HM, Chawes BL, Litonjua AA, et al. Prenatal vitamin D supplementation reduces risk of asthma/recurrent wheeze in early childhood: a combined analysis of two randomized controlled trials. PLoS One 2017; 12: e0186657.

7 Moffatt MF, Gut IG, Demenais F, et al. A large-scale, consortium-based genomewide association study of asthma. N Engl J Med 2010; 363: 1211-1221.

8 Torgerson DG, Ampleford EJ, Chiu GY, et al. Meta-analysis of genome-wide association studies of asthma in ethnically diverse North American populations. Nat Genet 2011; 43: 887-892.

9 Bisgaard H, Bonnelykke K, Sleiman PM, et al. Chromosome 17q21 gene variants are associated with asthma and exacerbations but not atopy in early childhood. Am J Respir Crit Care Med 2009; 179: 179-185.

10 Stokholm J, Chawes BL, Vissing N, et al. Cat exposure in early life decreases asthma risk from the 17q21 high-risk variant. J Allergy Clin Immunol 2018; 141: 1598-1606.

11 Hanada K. Serine palmitoyltransferase, a key enzyme of sphingolipid metabolism. Biochim Biophys Acta 2003; 1632: $16-30$.

12 Verlaan DJ, Berlivet S, Hunninghake GM, et al. Allele-specific chromatin remodeling in the ZPBP2/GSDMB/ ORMDL3 locus associated with the risk of asthma and autoimmune disease. Am J Hum Genet 2009; 85: 377-393.

13 Ono JG, Worgall TS, Worgall S. Airway reactivity and sphingolipids-implications for childhood asthma. Mol Cell Pediatr 2015; 2: 1-6.

14 Worgall TS, Veerappan A, Sung B, et al. Impaired sphingolipid synthesis in the respiratory tract induces airway hyperreactivity. Sci Transl Med 2013; 5: $186 \mathrm{ra} 167$.

15 Miller M, Broide DH. Why is ORMDL3 on chromosome 17q21 highly linked to asthma? Am J Respir Crit Care Med 2019; 199: 404-406.

16 Schmiedel BJ, Seumois G, Samaniego-Castruita D, et al. 17q21 asthma-risk variants switch CTCF binding and regulate IL-2 production by T cells. Nat Commun 2016; 7: 13426.

17 Sharma S, Zhou X, Thibault DM, et al. A genome-wide survey of CD4(+) lymphocyte regulatory genetic variants identifies novel asthma genes. J Allergy Clin Immunol 2014; 134: 1153-1162.

18 Seuter S, Neme A, Carlberg C. Characterization of genomic vitamin D receptor binding sites through chromatin looping and opening. PLoS One 2014; 9: e96184.

19 Litonjua AA, Lange NE, Carey VJ, et al. The Vitamin D Antenatal Asthma Reduction Trial (VDAART): rationale, design, and methods of a randomized, controlled trial of vitamin $\mathrm{D}$ supplementation in pregnancy for the primary prevention of asthma and allergies in children. Contemp Clin Trials 2014; 38: 37-50.

20 Bisgaard H. The Copenhagen Prospective Study on Asthma in Childhood (COPSAC): design, rationale, and baseline data from a longitudinal birth cohort study. Ann Allergy Asthma Immunol 2004; 93: 381-389.

21 Miller M, Rosenthal P, Beppu A, et al. ORMDL3 transgenic mice have increased airway remodeling and airway responsiveness characteristic of asthma. J Immunol 2014; 192: 3475-3487. 
22 Martineau AR, Cates CJ, Urashima M, et al. Vitamin D for the management of asthma. Cochrane Database Syst Rev 2016; 9: CD011511.

23 Breslow DK, Collins SR, Bodenmiller B, et al. Orm family proteins mediate sphingolipid homeostasis. Nature 2010; 463: 1048-1053.

24 Moffatt MF, Kabesch M, Liang L, et al. Genetic variants regulating ORMDL3 expression contribute to the risk of childhood asthma. Nature 2007; 448: 470-473.

25 Stein MM, Thompson EE, Schoettler N, et al. A decade of research on the 17q12-21 asthma locus: piecing together the puzzle. J Allergy Clin Immunol 2018; 142: 749-764.

26 Dautel SE, Kyle JE, Clair G, et al. Lipidomics reveals dramatic lipid compositional changes in the maturing postnatal lung. Sci Rep 2017; 7: 40555.

27 Magrassi L, Adorni L, Montorfano G, et al. Vitamin D metabolites activate the sphingomyelin pathway and induce death of glioblastoma cells. Acta Neurochir 1998; 140: 707-714.

28 Manggau M, Kim D-S, Ruwisch L, et al. 1 $\alpha, 25$-Dihydroxyvitamin $\mathrm{D}_{3}$ protects human keratinocytes from apoptosis by the formation of sphingosine-1-phosphate. J Invest Dermatol 2001; 117: 1241-1249.

29 Lester LA, Rich SS, Blumenthal MN, et al. Ethnic differences in asthma and associated phenotypes: Collaborative Study on the Genetics of Asthma. J Allergy Clin Immunol; 108: 357-362.

30 van der Velden JM, Verkooijen HM, Young-Afat DA, et al. The cohort multiple randomized controlled trial design: a valid and efficient alternative to pragmatic trials? Int J Epidemiol 2017; 46: 96-102.

31 Bisgaard H, Stokholm J, Chawes BL, et al. Fish oil-derived fatty acids in pregnancy and wheeze and asthma in offspring. N Engl J Med 2016; 375: 2530-2539.

32 Vesper H, Schmelz E-M, Nikolova-Karakashian MN, et al. Sphingolipids in food and the emerging importance of sphingolipids to nutrition. J Nutr 1999; 129: 1239-1250.

33 Babenko NA. Brain, fish oil-enriched diet, and sphingolipids. In: Raatz SK, Bibus DM, eds. Fish and Fish Oil in Health and Disease Prevention. San Diego, Academic Press, 2016; pp. 263-272.

34 Haddad SA, Ruiz-Narvaez EA, Cozier YC, et al. Association of degree of European genetic ancestry with serum vitamin D levels in African Americans. Am J Epidemiol 2018; 187: 1420-1423.

35 Worgall TS. Sphingolipids, ORMDL3 and asthma: what is the evidence? Curr Opin Clin Nutr Metab Care 2017; 20: 99-103.

36 Hollams EM, Hart PH, Holt BJ, et al. Vitamin D and atopy and asthma phenotypes in children: a longitudinal cohort study. Eur Respir J 2011; 38: 1320-1327.

37 Kho AT, Sharma S, Qiu W, et al. Vitamin D related genes in lung development and asthma pathogenesis. BMC Med Genomics 2013; 6: 47.

38 Zosky GR, Berry LJ, Elliot JG, et al. Vitamin D deficiency causes deficits in lung function and alters lung structure. Am J Respir Crit Care Med 2011; 183: 1336-1343.

39 Oyeniran C, Sturgill JL, Hait NC, et al. Aberrant ORM (yeast)-like protein isoform 3 (ORMDL3) expression dysregulates ceramide homeostasis in cells and ceramide exacerbates allergic asthma in mice. J Allergy Clin Immunol 2015; 136: 1035-1046.e1036. 